\title{
ANH
}

\section{ANSIEDADE E PERCEPÇÕES DE MORTE E MORRER ENTRE GRADUANDOS DE ENFERMAGEM}

\author{
Suélen Aparecida Costa ${ }^{1}$, Ivi Ribeiro Back ${ }^{2}$, Iven Giovanna Trindade Lino ${ }^{3}$, Verônica Francisqueti \\ Marquete $^{4}$, Maria Emília Grassi Busto Miguel $^{5}$, Sonia Silva Marcon ${ }^{6}$
}

\section{RESUMO}

Objetivo: Verificar o grau de ansiedade face à morte e as diferentes perspectivas do processo de morte e morrer entre graduandos do último ano de enfermagem.

Método: Estudo transversal, realizado em cinco universidades do Noroeste do Paraná. Os dados foram coletados entre agosto e outubro de 2017, mediante questionário autoaplicável abordando questões sociodemográficas e as escalas de ansiedade e perspectivas da morte. Os dados foram analisados com auxílio da estatística descritiva e inferencial.

Resultados: A maioria $(41,7 \%)$ dos 108 acadêmicos apontou discussão insuficiente desta temática em sala de aula. Foi observada correlação entre ansiedade e as seguintes perspectivas de morte: sentimentos de sofrimento e solidão ( $p=0,0275)$, abandono como sensação de culpa $(p=0,0177)$ e assombro de fracasso $(p=0,0262)$.

Conclusão: A ansiedade e a religião estiveram associadas a sentimentos perante a morte que devem ser melhor abordados entre futuros enfermeiros.

Descritores: Enfermagem; Morte; Estudantes de Enfermagem.

1. Suélen Aparecida Costa - Enfermeira. Residente em Urgência e Emergência na Universidade Estadual de Maringá. Maringá, PR, Brasil. E-mail: suelen. apcosta@yahoo.com.br. ORCID iD: https://orcid.org/0000-0003-2359-8394

2. Ivi Ribeiro Back - Enfermeira e Nutricionista. Doutora em Ciências da Saúde. Professora do Curso de Enfermagem do Centro Universitário de Maringá CESUMAR. Maringá, PR, Brasil. E-mail: iviback@hotmail.com. ORCID iD: https://orcid.org/0000-0002-7867-8343.

3. Iven Giovanna Trindade Lino - Enfermeira. Doutoranda em Enfermagem na Universidade Estadual de Maringá. Maringá, PR, Brasil. E-mail: iven_giovanna@hotmail.com. ORCID iD: https://orcid.org/0000-0003-0631-9971.

4. Verônica Francisqueti Marquete - Enfermeira. Mestranda em Enfermagem na Universidade Estadual de Maringá. Maringá, PR, Brasil. E-mail: veronicafrancisqueti@hotmail.com. ORCID iD: https://orcid.org/0000-0002-8070-6091

5. Maria Emília Grassi Busto Miguel - Enfermeira. Doutora em Enfermagem. Docente das disciplinas Enfermagem Fundamental I e II do Curso de Enfermagem da Universidade Estadual de Maringá. Maringá, PR, Brasil. E-mail: megbmiguel@uem.br. ORCID iD: https://orcid.org/0000-0002-2046-7009

6. Sonia Silva Marcon - Enfermeira. Doutora em Filosofia da Enfermagem. Docente da Graduação e Pós-Graduação em Enfermagem da Universidade Estadual de Maringá. Maringá, PR, Brasil. E-mail: soniasilva.marcon@gmail.com.ORCID iD https://orcid.org/0000-0002-6607-362X

\section{Artigo Original}

\section{Autor Correspondente:}

Sonia Silva Marcon

Instituição vinculada: Departamento de Enfermagem, Universidade Estadual de Maringá Endereço: Avenida Colombo, nº 5790, 87020-900, Maringá, Paraná, Brasil

E-mail: soniasilva.marcon@gmail.com

Telefone: (44) 99982-2884

\section{Como citar esse artigo}

COSRA, S. A. et al. Ansiedade e percepções de morte e morrer entre graduandos de enfermagem. Advances in Nursing and

Health, v. 1, p. 67-84, Londrina, 2019. 


\section{INTRODUÇÃO}

No Brasil, estimativas apontam que aproximadamente 1.264 .175 milhões de pessoas morrem a cada ano por diferentes causas[1]. Contudo, a morte continua sendo um assunto complexo, difícil de ser abordado, tanto na vida pessoal como no campo profissional[2].

Destaca-se que um dos deveres do profissional de enfermagem é promover a assistência de enfermagem, fundamentada na promoção da qualidade da vida, em todo o ciclo vital, desde o nascimento até a morte e o luto[3]. No entanto, mesmo fazendo parte do cotidiano desses profissionais, a morte ainda se apresenta como situação disparadora de reações e sentimentos negativos, tanto na vida pessoal como na profissional, o que pode interferir negativamente na qualidade da assistência prestada ao paciente e seus familiares diante da possibilidade de óbito[4].

Salienta-se que, durante a formação acadêmica, os alunos estão em um processo de desenvolvimento de seu perfil profissional, e tudo o que interfere neste processo pode trazer consequências para o futuro profissional. Sendo assim, na área da saúde, as dificuldades de compreender o processo de morte e morrer e o cuidado dispensado nesse momento podem ser exacerbados[5], já que a maioria das instituições de ensino aborda essa temática de forma fragmentada e deficitária, não destinando espaço para discussão de vivências que possam levar o acadêmico a alcançar uma compreensão mais clara a respeito da morte[2], o que exige competência técnica, conhecimento, raciocínio, percepção e sensibilidade[6]. O déficit na formação do enfermeiro para se defrontar com a morte de pacientes críticos interfere na qualidade da assistência prestada[7].

Um fator que pode contribuir para que essa formação não ocorra de modo pleno é o fato de que os próprios professores não possuem habilidades suficientes para trabalhar com esse tema, sendo comum se sentirem despreparados e/ou inseguros[8]. Logo, discutir a morte durante a graduação pode meIhor preparar os acadêmicos para esta vivência tão frequente na área da saúde, assim como reduzir o estresse e a ansiedade diante de situações que fragilizam emocionalmente o cliente, seus familiares e, por vezes, os membros da equipe de enfermagem[9-11].

Tais comportamentos, além de interferir negativamente na relação enfermeiro-pa- 
ciente, colocam os profissionais em situação de vulnerabilidade emocional, podendo culminar na ocorrência da Síndrome de Burnout[12]. Portanto, é substancial que as instituições de ensino preparem os futuros profissionais de saúde para lidar com o processo de morte/morrer no cotidiano da prática profissional, com abordagem sobre o tema durante todo o ciclo de formação[10,12-13].

Assim, o objetivo do estudo foi verificar o grau de ansiedade face à morte e as diferentes perspectivas do processo de morte e morrer entre graduandos do último ano de enfermagem.

\section{MÉTODO}

Estudo transversal não probabilístico, realizado na Região Noroeste do Estado do Paraná com acadêmicos do último ano do curso de enfermagem de cinco universidades, sendo três públicas e duas privadas. Os participantes foram selecionados por conveniência, sendo incluídos os estudantes presentes em sala de aula nos dias definidos para a coleta de dados e que, voluntariamente, concordaram em participar.

Os dados foram coletados entre agosto e outubro de 2017, por meio de questioná- rio autoaplicável constituído de duas partes: a primeira com questões sobre características sociodemográficas (sexo, idade, estado civil, cor/raça e religião) e a abordagem da temática durante a formação; a segunda englobou duas escalas: "Diversas Perspectivas da Morte", traduzida e validada para o português[14], constituída por 43 itens distribuídos em oito fatores: 1) Dor e solidão, que representa a morte como um momento de agonia, isolamento, angústia e solidão; 2) Além-vida de recompensa - aponta uma imagem da morte como uma nova vida, com satisfação, recompensa, felicidade e união com Deus; 3) Indiferença, que indica a morte como um fenômeno indiferente ao ser humano; 4) Desconhecido, apresenta a perspectiva da morte como incerteza, mistério e desconhecimento; 5) Abandono, em que se imagina a morte como abandono e um momento para se sentir culpado; 6) Coragem a morte é concebida como oportunidade para demostrar virtudes, como enfrentar o último teste de vida; 7) Fracasso - indica a morte como algo que impede a realização do potencial pessoal como a realização de sentido e objetivos de vida; 8) Fim natural - sugere a morte como algo que faz parte do ciclo natural da vida. As respostas são apresentadas em escala do tipo Likert de sete pontos, va- 
riando de $1=$ Discordo totalmente até $7=$ Concordo totalmente. A pontuação máxima possível é de 258 e a mínima de 43 pontos.

A escala "Ansiedade Face à Morte", também validada[14], contém 11 questões e respostas em escala do tipo Likert de cinco pontos, em que $1=$ totalmente em desacordo (absolutamente não) e 5 = totalmente de acordo (absolutamente sim), sendo a pontuação máxima de 55 pontos e a mínima de 11.

Os dados foram duplamente digitados em planilha no Excel e analisados no programaStatisticalAnalysis Software (SAS - versão 9.3). Foi realizada análise descritiva para a caracterização da população com uso de média e desvio-padrão. As análises de correlação entre ansiedade e as diversas perspectivas da morte foram testadas por meio do teste de Spearman. O teste de Wilcoxon foi utilizado para verificar hipóteses entre as variáveis quanto ao tipo de instituição e religião. Para todos os testes, considerou-se nível de significância de 95\% $(a=0,05)$.

O estudo respeitou normas nacionais e internacionais de pesquisas com seres humanos (Parecer $n^{\circ} 2.171 .120$ ). Os participantes assinaram o Termo de Consentimento Livre e Esclarecido.

\section{RESULTADO}

Participaram do estudo 108 acadêmicos, sendo 55 de instituições públicas e 53 de privadas, com idade entre 18 e 49 anos, média de 26 anos $( \pm 7,24)$. Observou-se predomínio de acadêmicos do sexo feminino $(85,2 \%)$, solteiros $(76,9 \%)$, brancos (75,9\%) e católicos $(65,7 \%)$. Só foi observada diferença significativa entre instituições públicas e privadas em relação ao sexo, pois, enquanto as públicas tinham 5,5\% de acadêmicos do sexo masculino, nas privadas eles eram $24,5 \%$, as quais também apresentaram maior proporção de casados $(28,3 \times$ $18,2 \%)$, não brancos ( $26,4 \times 21,8 \%)$ e evangélicos (26,4 x 20,0\%).

A maioria dos acadêmicos afirmou já ter vivenciado alguma experiência relacionada à morte $(89,8 \%)$, com diferença significativa entre instituições públicas e privadas. Observaram-se diferenças significativas entre as instituições (públicas $\mathrm{x}$ privadas) também entre o local da experiência e o sentir-se preparado frente à morte de um paciente (Tabela 1). 
A abordagem do tema morte $\mathrm{X}$ morrer durante a graduação foi referida pela maioria(75,0\%), sendo que alunos de instituições públicas afirmaram maior aprendizado sobre o assunto durante os estágios, enquanto

alunos de instituições privadas o adquiriram na teoria $(p=0,007)$. As demais questões relacionadas à abordagem do tema durante a graduação estão apresentadas na Tabela 1.

Tabela 1 - Vivências da morte e percepções referentes ao curso de enfermagem segundo estudantes. Maringá, PR, Brasil, 2017.

\section{QUESTÕES ABORDADAS}

\section{Pública $n(\%)$}

\section{Privada $n(\%)$}

\section{Total} n(\%)

\section{EXPERIÊNCIA COM A MORTE}

\section{EXPERIÊNCIA COM A MORTE}

\begin{abstract}
Sim
\end{abstract}
Não

\section{EXPERIÊNCIA COM A MORTE}

Familiar

Graduação

Outro

Nenhum

MEDO DA MORTE

$\operatorname{Sim}$

Não

PREPARADO FRENTE À

MORTE DE UM PACIENTE

Despreparado
Parcialmente preparado
Preparado

$\begin{array}{ccc}54(98,2) & 43(81,1) & 97(89,8) \\ 1(1,8) & 10(18,9) & 11(10,2)\end{array}$

$1(1,8) \quad 10(18,9) \quad 11(10,2)$

$\begin{array}{ccc}38(69,1) & 33(62,3) & 71(65,7) \\ 8(14,5) & 0(0,0) & 8(7,4) \\ 8(14,5) & 10(18,9) & 18(16,7) \\ 1(1,8) & 10(18,9) & 11(10,2)\end{array}$

$\begin{array}{lll}14(25,5) & 11(20,8) & 25(23,1) \\ 41(74,5) & 42(79,2) & 83(76,9)\end{array}$

$\begin{array}{ccc}3(5,5) & 1(1,9) & 4(3,7) \\ 37(67,3) & 18(34,0) & 55(50,9) \\ 15(27,3) & 34(64,2) & 49(45,4)\end{array}$




\section{QUESTÕES ABORDADAS}

$$
\begin{aligned}
& \text { Pública } \\
& \mathbf{n}(\%)
\end{aligned}
$$
Privada
n(\%)

Total

n(\%)

p-valor

COMUNICAR O ÓBITO A UM FAMILIAR

Sim

Não

$\begin{array}{lll}22(40,0) & 34(64,2) & 56(51,9) \\ 33(60,0) & 19(35,8) & 52(48,1)\end{array}$

\section{REFERENTE À GRADUAÇÃO}

\section{TEVE ESTE ASSUNTO NA GRADUAÇÃO}

Sim

$37(67,3)$

$44(83,0)$

$81(75,0)$

0,059

Não

$18(32,7)$

$9(17,0)$

$27(25,0)$

QUAL DISCIPLINA

Teórica

Estágio/Prática

$13(23,6)$

$28(52,8)$

$41(38,0)$

0,007

Nenhuma

$29(52,7)$

$16(30,2)$

$45(41,7)$

$13(23,6)$

$9(17,0)$

$22(20,4)$

QUAL DISCIPLINA ABORDA ESSE TEMA

Fundamentos de enfermagem

Saúde do adulto

Psicologia

Anatomia

Bioética

PERCEPÇÃO MUDOU APÓS

A MORTE DE PACIENTE

Sim

Não

$\begin{array}{ccc}4(7,3) & 5(9,4) & 9(8,3) \\ 11(20,0) & 6(11,3) & 17(15,7) \\ 15(27,3) & 19(35,8) & 34(31,5) \\ 6(10,9) & 7(13,2) & 13(12,0) \\ 6(10,9) & 7(13,2) & 13(12,0)\end{array}$

$32(58,2)$

$29(54,7)$

$61(56,5)$

0,717 


\section{QUESTÕES ABORDADAS}

Pública
n(\%)

Privada
n(\%)

\section{Total $n(\%)$}

p-valor

\section{O QUE FALTA NA FORMAÇÃO DO ENFERMEIRO}

Contato precoce com pacientes
Discussão do conteúdo na graduação
Experiências anteriores com o processo
Humanização nas experiências
Interação com outras ciências
como psicologia

$\begin{array}{ccc}1(1,8) & 4(7,5) & 5(4,6) \\ 29(52,7) & 16(30,2) & 45(41,7) \\ 7(12,7) & 9(17,0) & 16(14,8) \\ 10(18,2) & 17(32,1) & 27(25,0) \\ 8(14,5) & 7(13,2) & 15(13,9)\end{array}$

Em relação à escala "Diversas Perspecsentados em tabela). A média referente aos tivas da Morte", observou-se uma pontuação média de 141 pontos $( \pm 54,08)$ e mediana de 139 pontos. A pontuação mínima foi de 44 fatores "Além-vida de recompensa" e "Desconhecido" foi maior que as obtidas nos outros fatores (Tabela 2).

e a máxima de 258 pontos(dados não apre-

Tabela 2 - Fatores médios da escala "Diversas Perspectivas da Morte" entre estudantes de enfermagem. Maringá, PR, Brasil, 2017.

\section{DIVERSAS PERSPECTIVAS DA MORTE}

Dor e solidão

Além-vida de recompensa

Indiferença

Desconhecido

Abandono

Coragem

Fracasso

Fim natural
Média ( $($ )

\section{Valor Máx.}

Valor Mín. 
Já em relação à escala "Ansiedade Face à Morte",observou-se uma pontuação média de 38,74 pontos $( \pm 9,18)$ e mediana de 39 pontos. A pontuação mínima foi de 14 e a máxima de 55 pontos. Tais resultados refle- tem uma elevada tendência para ansiedade face à morte na amostra em estudo, visto que a pontuação máxima desta escala é de 55 pontos.

Tabela 3 - Perspectivas de morte e morrer de acadêmicos de enfermagem segundo tipo de instituição de ensino. Maringá, PR, Brasil, 2017.

\begin{tabular}{|c|c|c|c|}
\hline VARIÁVEIS & INSTITUIÇÃO & ESCORE MÉDIO & p-valor \\
\hline Dor e solidão & $\begin{array}{l}\text { Pública } \\
\text { Privada }\end{array}$ & $\begin{array}{l}51.2 \\
57.8\end{array}$ & 0.1346 \\
\hline Além-vida de recompensa & $\begin{array}{l}\text { Pública } \\
\text { Privada }\end{array}$ & $\begin{array}{l}54.9 \\
54.0\end{array}$ & 0.4377 \\
\hline Indiferença & $\begin{array}{l}\text { Pública } \\
\text { Privada }\end{array}$ & $\begin{array}{r}51.3 \\
57.8\end{array}$ & 0.1395 \\
\hline Desconhecido & $\begin{array}{l}\text { Pública } \\
\text { Privada }\end{array}$ & $\begin{array}{l}55.1 \\
53.8\end{array}$ & 0.4207 \\
\hline Abandono & $\begin{array}{l}\text { Pública } \\
\text { Privada }\end{array}$ & $\begin{array}{l}50.1 \\
58.9\end{array}$ & 0.0719 \\
\hline Coragem & $\begin{array}{l}\text { Pública } \\
\text { Privada }\end{array}$ & $\begin{array}{l}52.5 \\
56.5\end{array}$ & 0.2521 \\
\hline
\end{tabular}




$\begin{array}{llll} & \text { Pública } & 52.6 & 0.2615 \\ \text { Fracasso } & \text { Privada } & 56.4 & \\ & & & \\ \text { Fim natural } & \text { Pública } & 55.2 & 0.3960 \\ & \text { Privada } & 53.6 & \end{array}$

Ao investigar a associação entre diversas perspectivas de morte e religião, observou-se que os evangélicos acreditam que a morte seja um caminho para uma nova vida $(p=<0,005)$ (Tabela4).

Tabela 4 - Perspectivas de morte e morrer segundo a religião de acadêmicos de enfermagem. Maringá, PR, Brasil, 2017.

\section{VARIÁVEIS}

Dor e solidão

Dor solidão

Além-vida de recompensa

Alem-vida de recompensa

Indiferença

Desconhecido
RELIGĨ̃O

Católica

Evangélica

Outra/Nenhuma

Católica

Evangélica

Outra/Nenhuma

Católica

Evangélica

Outra/Nenhuma

Católica

Evangélica

Outra/Nenhuma
ESCORE MÉDIO

59,1

0,0759

48,3

39,8

56,6

0,0005

63,8

22,4

54,5

0,7484

51,7

60,0

57,2

0,1069

55,2

36,6 
VARIÁVEIS

RELIGIÃO

ESCORE MÉDIO

p-valor

\begin{tabular}{|c|c|c|c|}
\hline \multirow{3}{*}{ Abandono } & Católica & 55,3 & \multirow[t]{3}{*}{0,8009} \\
\hline & Evangélica & 54,8 & \\
\hline & Outra/Nenhuma & 48,8 & \\
\hline \multirow{3}{*}{ Coragem } & Católica & 55,4 & \multirow[t]{3}{*}{0,3721} \\
\hline & Evangélica & 57,5 & \\
\hline & Outra/Nenhuma & 42,7 & \\
\hline \multirow{3}{*}{ Fracasso } & Católica & 56,5 & \multirow[t]{3}{*}{0,4618} \\
\hline & Evangélica & 53,4 & \\
\hline & Outra/Nenhuma & 44,5 & \\
\hline \multirow{3}{*}{ Fim natural } & Católica & 56,1 & \multirow[t]{3}{*}{0,7016} \\
\hline & Evangélica & 52,3 & \\
\hline & Outra/Nenhuma & 48,9 & \\
\hline
\end{tabular}

Ao correlacionar ansiedade com as diversas perspectivas da morte, constataram-se valores significativos para as subes-

Tabela 5 - Correlação entre ansiedade e diversas perspectivas da morte entre alunos de enfermagem. Maringá, PR, Brasil, 2017.
ANSIEDADE(R)

calas: dor e solidão $(p=0,0275)$, abandono $(p=0,0177)$ e fracasso $(p=0,0262)$ (Tabela5).

p-valor

DIVERSAS PERSPECTIVAS DA MORTE

Dor e solidão

0,21217

0,0275

Além-vida de recompensa

0,11594

0,2321

Indiferença

0,06730

0,4889

Desconhecido

0,09643

0,3208

Abandono

0,22792

0,0177 
Coragem

Fracasso

Fim natural
0,06503

0,21391

0,12149
0,5037

0,0262

0,2104

\section{DISCUSSÃO}

Grande parte dos acadêmicos relatou já ter vivenciado experiências relacionadas com a morte, porém foi possível identificar diferença na abordagem desta temática entre estudantes de instituições públicas e privadas. Nas públicas, a abordagem ocorre com maior frequência em atividades práticas (estágios), enquanto nas privadas, isso ocorre em aulas teóricas. Constatou-se, ainda, correlação positiva entre ansiedade face à morte e as diversas perspectivas de morte, ou seja, quanto mais elevados os níveis de ansiedade, mais frequentes os sentimentos de dor, solidão, abandono e fracasso perante a morte.

A maioria dos acadêmicos em estudo já teve uma experiência relacionada com a morte. No entanto, ela foi, majoritariamente, em âmbito familiar. Ressalta-se que graduandos de instituições públicas tiveram mais contato com a morte de pacientes do que os de instituições privadas. Este resultado assemelha-se a de estudo realizado na Universidade de Brasília com 995 graduandos de cursos da área da saúde, no qual foi identificado que apenas $11,3 \%$ deles já tinham tido alguma experiência com a morte de paciente[15].

Tais dados indicam o pouco contato dos alunos com pacientes críticos, em fase terminal ou em salas de emergência nas situações práticas. Apesar disto, grande parte dos participantes afirmou não ter medo de presenciar o óbito de paciente, embora apenas pouco mais da metade sinta-se parcialmente preparada para esta situação. Nesta direção, estudo realizado com 50 acadêmicos de enfermagem verificou maior frequência de medo $(62,0 \%)$ em relação à morte de alguém em estudantes que se consideravam parcialmente preparados para a perda de um paciente [16]. 
Graduandos das instituições privadas demonstraram sentir-se mais preparados frente à morte de um paciente, o que provavelmente está relacionado ao fato de, segundo eles, esta temática ser abordada durante a graduação mais do que nas instituições públicas. Contudo, observou-se que pouco mais da metade dos participantes afirmou estar preparada para comunicar o óbito aos familiares dos pacientes. A literatura aponta que esta não é uma tarefa fácil, pois exige sensibilidade, paciência, empatia, conhecimento científico, entre outras habilidades. Mesmo profissionais com larga experiência, por vezes, sentem dificuldades em fazer este tipo de comunicado. Concernente a isto, é certo que, durante as atividades práticas de estágio, os acadêmicos podem até ter a oportunidade de acompanhar um profissional mais experiente na realização desta tarefa, mas jamais serão os responsáveis por ela durante a graduação[17]. Sendo assim, precisarão de um tempo após a graduação para adquirirem habilidades que Ihes permitam fazer este tipo de comunicado.

Para que o aluno finalize a graduação com preparo suficiente para enfrentar o processo de morte e morrer, este deve ser abordado suficientemente e seguindo suas diversas perspectivas, tanto no âmbito teórico quanto no prático. Porém, 20,4\% dos graduandos em estudo, apesar de estarem no último semestre do curso, afirmaram que o tema não foi abordado ao longo do curso.

Acredita-se que a abordagem deste tema de forma sistemática na disciplina de psicologia pode contribuir de forma efetiva para o preparo do futuro profissional. Nela é possível trabalhar a ansiedade dos alunos e prepará-los para o enfrentamento da situação de morte durante os estágios e na vida profissional e, inclusive, sua abordagem junto à família, quando necessário[18]. Contudo, devido à complexidade que envolve esta temática, isso tem que ser retomado em diversas disciplinas ao longo do curso. Inclusive, os docentes necessitam aproveitar a ocorrência de óbitos durantes os estágios clínicos para promover a discussão em grupo e possibilitar que os acadêmicos expressem seus sentimentos diante do ocorrido. Destaca-seque a assistência de enfermagem deve englobar informações éticas, psicológicas, históricas, religiosas e culturais[19].

Em relação às diferenças observadas quanto às perspectivas de morte nas diferentes religiões, constatou-se que entre os evangélicos a concepção de morte como 
"Além-vida de recompensa" foi mais frequente, ou seja, enxergam a morte como uma recompensa e união com Deus, enquanto que entre os católicos, apesar de maior destaque para "Dor e solidão", não foram observadas diferenças entre as diversas concepções sobre a morte e o morrer. Este resultado reforça que as crenças religiosas podem influenciar a maneira como a pessoa enfrenta as perdas. Concernente a isto, estudo realizado com mulheres que vivenciaram o processo de luto do cônjuge identificou que evangélicos possuem percepções diferentes em relação à morte quando comparados com fiéis de demais religiões[20].

Destarte, a religião é um relevante fator no processo de aceitação e enfrentamento da morte, pois as pessoas, muitas vezes, encontram nela forças para melhor tolerar essa fase difícil[21]. Ademais, independentemente da crença, possuir algum envolvimento religioso faz com que as pessoas sintam menos medo da morte, pois se amparam nos ensinamentos religiosos para encontrar respostas sobre os porquês da vida e da morte e o que acontece após a morte[22].

Nesta direção, estudo com enfermeiros evidenciou que em suas percepções há meIhor aceitação da iminência da morte e do sofrimento nos pacientes religiosos; e que, diante da possibilidade da finitude, os pacientes se apegam mais à religião[23].

Em relação à ansiedade, os dados apontaram que os acadêmicos possuíam elevado grau de ansiedade face à morte (escore médio de 38,7$)$. Estudo realizado em Portugal identificou elevado nível de ansiedade entre os profissionais de saúde, porém com escore médio mais baixo $(33,9)(24)$. Grau mais elevado de ansiedade entre os acadêmicos pode ser explicado pela falta de experiência associada à pouca abordagem do tema ao longo do curso.

Verificou-se que quanto maior a ansiedade face à morte, maior a frequência de sentimentos negativos caracterizados por "Dor e solidão", "Sensação de abandono", de "Culpa por deixar seus entes queridos" e de "Assombro de fracasso". Estudo realizado em Campina Grande-PB com 400 estudantes de psicologia, enfermagem e medicina verificou que o medo mais comum entre eles foi o de ter uma morte dolorosa e/ou de vivenciar o processo de morrer aos cuidados de pessoas desconhecidas[25].

Para auxiliar nesse enfrentamento, as estratégias de ensino utilizadas devem permitir que os graduandos encontrem apoio e 
segurança, atualizem seus conhecimentos sobre a causa clínica do óbito e ofereçam suporte aos familiares e pacientes envolvidos no processo de morte/morrer[26]. Dentre as ações possíveis, destaca-se a necessidade e importância da integração da equipe multiprofissional com discussões práticas dos casos entre todos os profissionais, tendo em vista a melhoria da qualidade do enfretamento perante o processo de finitude da vida[13].

Desse modo, discutir a formação dos enfermeiros diante do processo de morte e morrer assume importância quando se trata de amenizar os anseios, ansiedade e comprometimento da saúde mental dos profissionais de saúde, devido às dificuldades para prestar uma assistência de qualidade e humanizada aos pacientes e familiares. Urge introduzir na formação acadêmica uma visão crítico-reflexiva sobre o processo de morte e morrer, pois a prestação de cuidados de qualidade a pessoas em situação de finitude da vida é possível, especialmente quando se está preparado para esse fim[10].

Estudo que realizou intervenção com estudantes de enfermagem em Macaé-RJ, utilizando um método participativo de ensino-aprendizagem, sobre o tema da morte, morrer e cuidados associados, visando fa- vorecer a problematização da temática, verificou que, ao exporem as próprias realidades, sentimentos, medos e angústias sobre o tema, por meio do desenho de cenas hipotéticas nas quais eram oferecidos cuidados a pacientes em processo de morte, os estudantes puderam refletir, criticar ou alterar cenas, o que, metaforicamente, se traduziu em rever posturas, interpretar situações e atribuir novos significados, adquirindo saberes e reconhecendo a necessidade de buscar novos conhecimentos para lidar com esse processo[6].

Estudo realizado no Canadá aponta que o setor de pediatria do hospital universitário utiliza como estratégias de enfrentamento discussões sobre morte/morrer com os pacientes e seus pais, e entre profissionais de saúde, onde a maioria dos conflitos é frequentemente solucionada no momento da morte. Os profissionais também destacam a importância de reuniões interdisciplinares em duas etapas - entre os profissionais e os pais - reuniões de ética pós-morte, protocolos de acompanhamento de luto e serviços de ética clínica[27]. Por fim, outras estratégias apontadas na literatura para favorecer o desenvolvimento de habilidades e atuação em situações de enfrentamento da morte e morrer no cotidiano profissional são os jogos 
experimentais[28] e os grupos de educação para a morte, que são desenvolvidos como atividades extracurriculares[29].

Possíveis limitações do estudo estão relacionadas ao delineamento adotado, pois estudos transversais não permitem observar a relação de causa e efeito (morte e morrer $\mathrm{X}$ ansiedade entre os alunos) e também ao fato de que a maioria dos participantes é do sexo feminino, o que não permitiu identificar se existe diferença de perspectivas e de ansiedade entre homens e mulheres. De qualquer modo, seus resultados são válidos, pois ainda são escassos estudos sobre o grau de ansiedade face à morte e sobre as diferentes perspectivas do processo de morte e morrer entre acadêmicos de enfermagem, apesar de sua importância, visto que no futuro enfrentarão com frequência estas situações. Assim, aponta-se como necessária a desmistificação da morte e do processo de morrer ainda durante a graduação. Por conseguinte, este estudo contribui na medida em que seus resultados mostram aspectos importantes relacionados a esta problemática.

\section{CONCLUSÃO}

Os resultados permitem concluir que os acadêmicos do último ano dos cursos de enfermagem em estudo apresentam elevado grau de ansiedade face à morte e que a maioria já vivenciou alguma experiência relacionada à morte, especialmente no âmbito familiar. Apesar de considerar haver uma lacuna na abordagem do assunto na graduação, boa parte dos acadêmicos se autodeclarou preparada para enfrentar este processo, denotando que eles podem estar banalizando este preparo, pois foi apresentado elevado grau de ansiedade frente à morte, o que, por sua vez,pode ser explicado pela falta de experiência e pela pouca abordagem do tema ao longo do curso. 


\section{REFERÊNCIAS}

1. Ministério da Saúde (BR). Sistema de informação sobre mortalidade. DATASUS. Brasília, DF: Ministério da Saúde; 2015.

2. Bandeira D, Cogo SB, Hildebrandt LM, Badke MR. A morte e o morrer no processo de formação de enfermeiros sob a ótica de docentes de enfermagem. Texto Contexto Enferm. 2014jun; 23(2):400-07. doi: http://dx.doi. org/10.1590/0104-07072014000660013

3. Conselho Federal de Enfermagem (COFEN). Resolução Cofen 564/2017. Código de ética dos profissionais de enfermagem [Internet]. Brasília, DF: COFEN; 2017. [citado 2019 jun. 20]. Disponível em: http://www. cofen.gov.br/wp-content/uploads/2017/12/ RESOLU \% C 3 \% $87 \%$ C $3 \% 830$ - COFEN N\%C2\%BA-564-2017.pdf.

4. Nunes NLF, Araújo KM, Silva LDC. As evidências sobre o impacto psicossocial de profissionais de enfermagem frente à morte. Rev Interd [Internet]. 2016 [citado 2019jun. 22];9(4):165-72. Disponível em: https:// revistainterdisciplinar.uninovafapi.edu.br/ index.php/revinter/article/view/545.

5. Oliveira ES, Agra G, Morais MF, Feitosa IP, Gouveia BLA, Costa MML. The process of death and dying in nursing students perception. J Nurs UFPE on line. 2016 May;10(5):170916. doi: 10.5205/reuol.9003-78704-1SM.1005201617

6. Lima R, Bergold LB, Souza JDF, Barbosa GS, Ferreira MA. Death education: sensibility for caregiving. Rev Bras Enferm. 2018;71(suppl 4):1884-89. doi: 10.1590/0034-7167-20170018
7. Freitas TLL, Banazeski AC, Eisele A, Souza EM, Bitencourt JVOV, Souza SS. The look of nursing on death and dying process of critically ill patients: an integrative review. Enferm Glob [Internet]. 2016 [cited 2019 Aug 21];15(41):322-34. Available from: http:// scielo.isciii.es/pdf/eg/v15n41/en_revision2. pdf

8. Nunes ECDA, Santos AA. Challenges of nursing teaching-learning to care for human dying - professors' perceptions. Esc Anna Nery. 20170ct;21(4):e20170091. doi: 10.1590/2177-9465-EAN-2017-0091

9. Benedetti GMS, Oliveira K, Oliveira WT, Sales CA, Ferreira PC. Meaning of the death / dying process for entering nursing students. Rev Gaúcha Enferm. 2013 Mar;34(1):173-79. doi: 10.1590/S1983-14472013000100022

10. Jafari M, Rafiei $H$, Nassehi A, Soleimani $F$, Arab M, Noormohammadi MR. Caring for dying patients: attitude of nursing students and effects of education. Indian J Palliat Care. 2015 Aug;21(2):192-97. doi: 10.4103 / 0973-1075.156497.

11. Lima BSF, Silva RCL. Death and dying in a pediatric ICU: challenges for nursing care in the finitude of life. Cienc Cuid Saude. 2014 Out/Dez;13(4):722-29. doi: 10.4025/ cienccuidsaude.v13i4.21530

12. Santos MAD, Hormanez, M. Atitude frente à morte em profissionais e estudantes de enfermagem: revisão da produção científica da última década. Ciênc Saúde Coletiva. 2013 set;18(9):2757-768. doi: http://dx.doi. org/10.1590/S1413-81232013000900031.

13. Ferreira JMG, Nascimento JL, Sa FC. Profissionais de saúde: um ponto de vista sobre a morte e a distanásia. Rev 
Bras Educ Med. 2018;42 (3):87-96. doi: 10.1590/1981-52712015v42n3rb20170134

14. Barros-Oliveira J, Neto F. Validação de um instrumento sobre diversas perspectivas da morte. Aná Psicol [Internet]. 2004 [citado 2019 ago. 20];22(2):355-67. Disponível em: http://www.scielo.mec.pt/pdf/aps/v22n2/ v22n2a04.pdf

15. Ribeiro $\mathrm{HJ}$. Atitude e ansiedade face à morte: perfil dos graduandos da área da saúde [Monografia]. Brasília (DF): Universidade de Brasília; 2014.

16. Borges MC, Gomes BLBD. Percepção dos acadêmicos de enfermagem quanto ao processo terminal da vida. Rev Enferm Contemp. 2017 abr;6(1):49-55. doi: 10.17267/2317-3378rec.v6i1.1183

17. Duarte AC, Almeida DV, Popin RC. Death within the medical undergraduate routine: students' views. Interface (Botucatu). 2015; 9(55):1207-19. doi: 10.1590/180757622014.1093

18. Mendonça GA, Souza Junior VD, Correio NLS, Santos AS. O morrer para graduandos em enfermagem: a contribuição da psicologia. REFACS [Internet]. 2013;1(1):24-33 [citado 2019 ago. 20]. Disponível em: http://seer. uftm.edu.br/revistaeletronica/index.php/ refacs/article/view/492/pdf.

19. Custódio MRM. O processo de morte e morrer no enfoque dos acadêmicos de enfermagem. Rev Psicol [Internet]. 2010 [citado 2019 ago. 20];13(18):127-42. Disponível em: https:// revista.pgsskroton.com.br/index.php/renc/ article/view/2538/2425

20. Farinasso ALC, Labate RC. Luto, religiosidade e espiritualidade: um estudo clínico-qualitativo com viúvas idosas. Rev Eletr Enf [Internet]. 2012set;1(1):588-95 [citado 2019 ago. 23]. Disponível em: https://www.fen.ufg.br/ revista/v14/n3/pdf/v14n3a15.pdf

21. Bousso RS, Poles K, Serafim TS, Miranda MG. Religious beliefs, illness and death: family's perspectives in illness experience. Rev Esc Enferm USP. 2011 Apr; 45(2):397-403. doi:10.1590/S0080-62342011000200014

22. Barbosa AMGC, Massaroni L. Living with death and dying. J Nurs UFPE on line. 2016 Feb; 10(2):457-63 doi: 10.5205/reuol.855774661-1-SM1002201611

23. Silva $C R$, Portella $S D C$, Reis $L R$, Bispo TCF. Religião e morte: qual a relação existente. Rev Enferm Contemp. 2012;1(1):130-41. doi:10.17267/2317-3378rec.v1i1.49

24. Campelos ICSF. A ansiedade e o medo da morte nos profissionais de saúde [Monografia]. Porto (POR): Universidade Fernando Pessoa; 2006.

25. Ribeiro DB, Fortes RC. A morte e o morrer na perspectiva de estudantes de enfermagem. Revisa [Internet]. 2012 [citado 2019 ago. 10];1(1):32-9. Disponível em: http:// revistafacesa.senaaires.com.br/index.php/ revisa/article/view/10/7

26. Stochero HM, Nietsche EA, Salbego C, Pivetta $A$, Schwertner MVE, Fettermann FA, et al. Sentimentos e dificuldades no enfrentamento do processo de morrer e de morte por graduandos de enfermagem. Aquichan. 2016 jun;16(2):219-20. doi: 10.5294/ aqui.2016.16.2.9

27. Archambault-Grenier MA, Roy-Gagnon $M H_{\text {, }}$ Gauvin F, Doucet H, Humbert N, Stojanovic S, et al. Teamwork and conflicts in paediatric end of life care. Acta Paediatr. 2018 Feb;107(2):26269. doi: https://doi.org/10.1111/apa.14102 
28. Lopez Alonso AI, Fernández Martínez ME, Liébana Presa C, Vasquez Casares AM, Castro González MP. Experimental classroom games: a didactic tool in palliative care. Rev Esc Enferm USP. 2018 Apr; 52:e03310. doi: http://dx.doi. org/10.1590/S1980-220X2017007703310

29. Oliveira-Cardoso EA, Santos MA. Grupo de educação para a morte: uma estratégia complementar à formação acadêmica do profissional de saúde. Psicol Ciênc Prof. 2017;37(2):500-14. doi: http://doi. org/10.1590/1982-3703002792015 